\title{
La participation habitante dans les écoquartiers, un enjeu de (re)politisation?
}

Resident participation, bringing politics back to ecodistricts?

\section{Pascal Tozzi, Abdourahmane Ndiaye et Aurélie Carimentrand}

\section{(2) OpenEdition}

\section{Édition électronique}

URL : http://journals.openedition.org/developpementdurable/10913

DOI : 10.4000/developpementdurable.10913

ISSN : 1772-9971

Éditeur

Association DD\&T

\section{Référence électronique}

Pascal Tozzi, Abdourahmane Ndiaye et Aurélie Carimentrand, «La participation habitante dans les écoquartiers, un enjeu de (re)politisation?», Développement durable et territoires [En ligne], Vol. 6, n 2 | Septembre 2015, mis en ligne le 30 septembre 2015, consulté le 19 avril 2019. URL : http:// journals.openedition.org/developpementdurable/10913; DOI : 10.4000/developpementdurable.10913

Ce document a été généré automatiquement le 19 avril 2019.

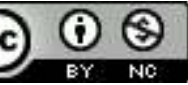

Développement Durable et Territoires est mis à disposition selon les termes de la licence Creative Commons Attribution - Pas d'Utilisation Commerciale 4.0 International. 


\title{
La participation habitante dans les écoquartiers, un enjeu de (re)politisation?
}

\author{
Resident participation, bringing politics back to ecodistricts?
}

Pascal Tozzi, Abdourahmane Ndiaye et Aurélie Carimentrand

Les coordinateurs tiennent à remercier Valérie Deldreve ainsi que l'ensemble des évaluateur(trice)s et relecteur(trice)s sans qui ce dossier n'aurait pu être mené à bien.

Compte tenu des nombreuses publications relatives à la question des écoquartiers, en France et ailleurs, ouvrir un nouvel espace de discussion scientifique sur le sujet nécessitait le choix d'un angle, sinon nouveau, tout au moins susceptible d'apports ou de discussions complémentaires. À ce titre, les relations entre processus participatifs et opérations de quartiers durables, bien qu'ayant été interrogées par les chercheur(e)s, sont loin d'avoir livré toute leur substance heuristique. Si les démarches participatives sont désormais un levier incontournable pour chaque acteur impliqué dans la gouvernance du projet urbain durable, les (re)mises en ordres ou en « désordres » qu'elles suscitent restent des processus centraux et complexes (Leresche et Audétat, 2006; Emelianoff 2008; Mathieu et Guermond 2011).

Dans ces démarches participatives et pour les parties prenantes à la fabrique de la ville durable, la qualité du processus d'élaboration va de pair avec la capacité d'y associer les habitants-citoyens et autres acteurs de la société civile. Elle devient un argument dans la valorisation des opérations écoquartiers et un critère convoqué pour les différencier des quartiers «classiques ». Grâce aux différentes modalités participatives, l'injonction vise pêle-mêle à éviter l'exclusion et la disqualification sociale de certaines populations; à obtenir un gain qualitatif dans la définition et l'appropriation des projets ; à réintégrer des références aux valeurs, à la citoyenneté et à de nouvelles solidarités urbaines, sociales et environnementales (Schaeffer, Ruegg et Litzistorf-Spina, 2010). L'objectif serait aussi de retisser des liens, des sociabilités, de développer durablement le tissu social et les identités locales. Pourtant, une fois cela posé en principe et en discours, le problème reste 
le passage de l'injonction de durabilité urbaine à la réalité d'une dynamique sociétale qui favoriserait la prise en charge collective, participative et pérenne, du cadre de vie (Bonard et Matthey, 2010). C'est ce discours participatif, ses effets et les expériences qu'il suscite (de même que ses dissonances), que les auteur(e)s réuni(e)s proposent d'approcher autour du présent dossier thématique intitulé «La participation habitante dans les écoquartiers : discours, effets, expérimentations et retours critiques", et à travers l'analyse concrète de processus de mise en durabilité et en écoquartiers de l'espace urbain.

3 Sans chercher à stabiliser l'écoquartier dans son contenu définitoire, l'éclairage multifocal et pluridisciplinaire qui ressort des présentes contributions permet néanmoins, par approximations et comparaisons successives, de mesurer la nébulosité de cet objet "quartier durable » et sa "viscosité »: entre "solide» et "fluide», entre l'emblématique et le diffus, entre les entreprises de modélisation et l'incertitude/ singularité des processus sociaux à l'œuvre. Quels que soient la diversité des échelles territoriales ou des types d'opérations sélectionnées, l'ensemble des articles rappellent la plupart des bornages techniques et opérationnels qui identifient l'écoquartier, au moins aux yeux de leurs promoteurs institutionnels : les caractéristiques « dures » du bâti, plus dense, plus compact, mieux isolé, moins gourmand en énergie et privilégiant ses sources renouvelables; la préoccupation pour les modes de transports doux, la prégnance des éco-technologies et des éco-normes, la promotion des espaces verts, etc. Parmi les prescriptions affichées, plus mouvantes et sujettes à diffraction, les auteur(e)s font aussi l'inventaire de la plupart des préconisations sociales qui font partie du package "écoquartier " pour les aménageurs, à l'instar de la construction des mixités, de la question des usages ou des attendus comportementaux dits "éco-citoyens ", et bien évidemment des formes de l'implication habitante, objet de ce dossier.

4 Le regard que portent les contributeurs(trices) sur la participation des habitants confirme son caractère centripète dans le discours, mais éminemment centrifuge dans les expérimentations et les dispositifs auxquels elle donne naissance, plus ou moins ouverts à la prise de décision habitante. Au-delà de retrouver, sur leurs terrains, certains invariants participatifs déjà mis en évidence par la littérature scientifique, les chercheur(e)s réuni (e)s dans ce dossier reposent, au prisme des écoquartiers et à travers les débats originaux qu'organise la rencontre de leurs contributions, la question cruciale des rapports entre participation et politique, notamment sur celle d'une politisation protéiforme des enjeux urbains dans la ville durable.

\section{Politisation institutionnelle et effets sur la participation des habitants d'écoquartiers}

$5 \mathrm{Au}$ fil de rappels historiques autant que législatifs ou règlementaires, les articles rappellent, dans leur ensemble, le double processus qui traverse l'aménagement des écoquartiers: l'institutionnalisation politique et administrative de la participation, combinée à celle de la durabilité. Cette dimension institutionnelle est d'autant plus forte que les registres de l'aménagement urbain durable s'hybrident à d'autres registres classiques et transversaux des politiques publiques: ville, logement, emploi, santé, vieillissement, propriété, etc. Autour de la ville durable et de ses adaptations participatives, les présentes contributions confirment ainsi la prégnance d'une action publique, parfois au détriment des mouvements «spontanés» de la société civile. Car 
même s'il se nourrit largement d'une volonté affichée de "partir de la base", le traitement de la participation - comme celui de la durabilité urbaine - confirme, au fil des articles proposés, qu'il se réalise dans des processus plutôt top down que bottom up. Les terrains investigués par les auteur(e)s, qu'il s'agisse d'opérations d'écoquartiers à Bordeaux, Lyon, Rennes, Poitiers, Paris, Toulouse, Strasbourg, Lille ou ailleurs, montrent ainsi que la conception en vigueur reste celle d'un "gouvernement de la participation " qui traverse les échelles spatiales de l'action aménageuse (Gourgues, 2013). Ce gouvernement se caractérise notamment par des cadrages forts dans le pilotage des opérations de quartiers durables en vue d'une conformation aux priorités politiques et aménageuses globales. Cela ne veut pas dire pour autant que la volonté des autorités publiques et aménageuses (promoteurs, bailleurs, etc.) n'aille pas dans le sens d'une implication des habitants (notamment pour des motifs stratégiques d'acceptabilité ou de légitimité), mais bien qu'elle reste surdéterminante et directive quant aux possibilités d'action et d'intégration offertes à ces derniers.

6 Concomitamment au pilotage de la fabrique participative de la ville durable par des dispositifs institutionnels et de politiques publiques, plusieurs études de cas montrent que les mécanismes d'une politisation descendante combinent aussi des stratégies de " décharge » et de « recyclage ». La gouvernance de certaines opérations donne ainsi à voir des situations où les autorités locales délèguent partiellement l'organisation de la participation, ou de la concertation, au profit de groupes d'habitants organisés. Pour les municipalités, cela permet notamment une externalisation des risques de conflictualité, mais aussi un « recyclage » des dynamiques participatives traditionnellement portées par ces secteurs associatifs qui jouent un rôle routinier d'interface entre les habitants, le projet politique et les adaptations urbaines. C'est ce que montrent Pascal Tozzi et Luc Greffier dans leur analyse de l'implication participative des centres sociaux et socioculturels partie-prenantes à certaines opérations d'écoquartiers bordelais. Au niveau national, cette politisation par recyclage, se retrouve dans le propos d'Abdourahmane Ndiaye, Aurélie Carimentrand et Marie-Reine Gallard lorsqu'ils détaillent le processus de reconnaissance étatique de nouvelles formes d'accès au logement par l'habitat participatif, qui connaît un début d'institutionnalisation au travers de la loi ALUR de 2014.

7 Ces processus de pilotage de l'action aménageuse ont à la fois un effet d'incitation et de restriction qui est relevé par plusieurs contributions. En effet, dans les processus participatifs institutionnels, tout n'est pas discuté, discutable ou remis en jeu devant les habitants et les associations locales. De même, la place faite, ou laissée, aux habitants révèle des périmètres mouvants et à géométrie variable, parfois entre les différentes phases d'un même projet d'écoquartier et selon les enjeux. Cette place s'avère souvent limitée et peut conduire à transformer les dispositifs participatifs en "théâtres de la participation, aboutissant à la construction de châteaux de cartes urbains " (Hélène Chelzen et Anne Jégou). Elle s'avère parfois plus importante (Nicolas D’Andréa et Florence Zérillo) sans empêcher quelques discontinuités (Camille Gardesse et Isabelle Grudet). Quelquefois, enfin, la sollicitation participative se montre risquée pour les collectifs d'habitants ou associatifs qui se voient confrontés à des processus d'instrumentalisation ou de «professionnalisation » n'allant pas sans ambiguïtés (Pascal Tozzi et Luc Greffier). Pour expliquer les contours variables de cette géométrie participative institutionnelle, les auteur(e)s évoquent parmi d'autres facteurs, les objectifs de rentabilité ou de sécurité financière qui hypothèquent par exemple la mise à disposition du foncier pour un projet 
d'habitat participatif (Camille Devaux), la persistance d'une méfiance du politique à l'égard des choix habitants notamment en matière architecturale (Camille Gardesse et Isabelle Grudet; Camille Devaux), l'invocation du caractère chronophage ou énergivore du processus participatif pour les porteurs de projet (Mathieu Adam, Georges Henry Laffont et Laura Seguin). D'un autre côté, les espaces participatifs peuvent exister et ne pas être occupés. Cela peut être par manque d'intérêt des habitants, par défiance et incrédulité vis-à-vis des décideurs publics et des instances de démocratie locale qu'ils animent (Guy Di Méo et Karen Foussette), ou encore à cause d'un investissement concurrent dans d'autres modalités de mobilisation habitante (Guillaume Faburel). Globalement, l'ensemble des auteur(e)s porte un regard critique sur les dispositifs institutionnels de la participation, avec leurs «zones à risque »classiques: manque d'ambition et de conviction participative, registres d'inaction et absence de réel pouvoir décisionnaire des habitants en dehors de micro-enjeux, complexité et dispersion des systèmes d'acteurs et des dispositifs, monopole ou captations de la parole légitime par les experts (habitants ou non), échec à inclure les publics fragilisés, tiers-absents et pourtant concernés par le projet d'écoquartier, impensé de la pérennisation d'une participation après livraison du quartier, incompatibilités des temporalités de l'organisation et de la mobilisation habitante avec celles du projet, faiblesse de la prise en compte des « savoirs d'usage », etc.

\section{La participation habitante dans les écoquartiers, entre dépolitisation et repolitisation}

8 Une fois relevée cette institutionnalisation politique de la participation et ses limites, certains auteurs présents dans ce dossier, posent chacun à leur manière, la question d'une possible dépolitisation, directe ou incidente, des processus participatifs - voire à cause d'eux - à l'échelle des écoquartiers. Le premier argument à l'appui de cette dépolitisation est celui d'une domestication de la participation habitante, donc d'une perte de la dimension conflictuelle et "sauvage " qui serait à même de lui conférer un caractère éminemment politique (Mermet, 2007). La deuxième objection est celle d'une limitation, voire d'une individuation « négative » des enjeux, qui minerait la puissance collective des revendications habitantes en empêchant une montée en généralité constitutive de tout processus de politisation. La participation institutionnalisée réduirait ainsi volontairement la possibilité de s'extraire des situations immédiates et donc de se référer à l'intérêt général. Dans cette perspective, Mathieu Adam, Georges Henry Laffont et Laura Seguin expliquent en quoi, selon eux, la conversion des contestations sociales et environnementales des années 1970 en injonctions de démocratie participative et de durabilité urbaine procède, aujourd'hui, d'une "trahison » des dimensions critiques et politiques originelles. Pour ces trois contributeurs, la planification actuelle de la participation et de la durabilité procède, dans ses discours et ses pratiques, d'une logique néolibérale et dépolitisante. Autour des écoquartiers, l'institutionnalisation participative hériterait d'une tendance à diluer la conflictualité au profit de formes contrôlées et acceptables de l'expression habitante. On se contenterait désormais de créer des «petits lieux » de démocratie (Bertheleu et Neveu, 2005) qui n'invitent pas à la montée en généralité, ni "à changer la ville pour changer la société ", mais qui se cantonnent à traiter de la proximité, de revendications ponctuelles surtout liées à des conflits d'usage. C'est aussi en ce sens que Didier Laugaa et Grégoire Le Campion relèvent, chez les 
habitants d'écoquartiers enquêtés, la tendance "clairvoyante " d'une conformation à l'ordre social existant (plutôt qu'à un positionnement conflictuel et militant) ainsi que, sans doute, à une norme consumériste. Ils évoquent à leur tour le risque que «la centration sur une participation au niveau du quartier diminue d'autant la participation à un niveau plus général et qu'elle se limite, alors, à une démocratie de proximitê" » réductrice car cantonnée à une échelle et à des enjeux micro-locaux.

Le débat se poursuit à travers d'autres contributions qui optent plutôt pour la persistance d'une politisation, ou pour la piste d'une repolitisation au sens d'une émergence de nouvelles formes d'une participation politique, en dehors des sentiers balisés par les institutions politico-administratives. En ce sens, s'ils posent la question de l'effet des évolutions récentes de la législation française, Abdourahmane Ndiaye, Aurélie Carimentrand et Marie-Reine Gallard persistent à penser que la promotion institutionnalisée de l'habitat participatif témoigne d'une forme de reconnaissance politique intégrant certes les problématiques environnementales, mais donnant surtout suite aux revendications sociales de solidarité et à la critique militante des inégalités sociales. Poursuivant le questionnement du rapport au politique, Guillaume Faburel croise quant à lui l'analyse de discours d'acteurs des projets avec l'observation de quatre écoquartiers étrangers, ainsi qu'avec trois français d'initiative habitante et/ou d'orientation sociale. Il propose d'appréhender la participation par le truchement des modes de vie qui procèderaient, selon lui, de formes d'engagement individuel désaffiliées (dont parlent aussi Mathieu Adam, Georges Henry Laffont et Laura Seguin), en parallèle, voire en réaction aux cadres institués de la participation officielle et traditionnelle de projet. Ainsi, les pratiques sociales à l'œuvre dans les écoquartiers seraient certes marquées par une individuation, mais qui ne vaudrait ni individualisation, ni dépolitisation : n'effaçant pas systématiquement la lutte politique ou la conflictualité, la résistance et la critique sociale, elles traduiraient plutôt une réorientation de l'engagement politique vers l'action « directe».

Pour Guillaume Faburel, le processus d'individuation participante se nourrit par exemple d'un rapport multisensoriel au paysage et au lieu qui procède des logiques d'appropriation et d'identification, elles-mêmes susceptibles de générer des expériences à la fois individuelles et collectivement partagées, donc de nouvelles formes d'intervention et d'implication dans l'espace public. L'" empaysagement » rimerait finalement avec " empowerment». Le projet politique participatif à l'œuvre dans les écoquartiers se dévoilerait alors comme celui d'un habiter porteur d'une dimension "infrapolitique " (Scott, 2006) et conservant toute la dimension collective à la participation, puisque articulant des valeurs et des actions faisant «commun ». Dans cette même idée d'une construction de l'individualité comme contribution à l'élaboration des finalités du groupe, Guy Di Meo et Karen Foussette proposent d'intégrer une dimension physique et expérientielle dans le rapport à l'urbain durable et à la participation. Ces auteurs posent la question d'une politique du corps pratiquant la ville. Ainsi, les usages subjectifs et subjectivants d'un corps-interface avec la ville, les corporéités spatiales avec leurs exigences de transparence urbaine (désir de justice sociospatiale et environnementale, de sécurité et de civilité, de nature), polariseraient les enjeux politiques et participatifs de la ville durable. Pour Guy Di Meo et Karen Foussette, les demandes très pragmatiques d'aménagement qui surgissent des besoins d'un corps individuel épris de bien-être, immergé dans l'espace urbain et demandeur d'interactions sociospatiales, sont peut-être le "véritable terreau politique de la ville durable, telle que vécue par ses habitants". 
C'est aussi ce type de perspectives que recèle, en filigrane, le texte de Nicolas d'Andréa et Florence Zérillo qui traite des problématiques d'une adaptation urbaine participative et durable aux corps vieillissants et aux nombreux remaniements psychiques, sociaux et biologiques, mais aussi de « déprise » sociospatiale, que le vieillissement induit.

Au final, des contributions réunies, la participation des habitants d'écoquartiers ressort notamment comme un processus en tension, entre participation institutionnalisée et participation désaffiliée, entre niveaux individuels et collectifs. Les différents textes montrent aussi que cette tension, bien que combinée à un certain désenchantement à l'égard de «la» politique et de la participation institutionnelles, n'entrâne pas forcément une crise » du » politique au sens large ni de la citoyenneté. Entre persistances, concurrences et évolutions des formes, se confirme le caractère dynamique et combinatoire du politique dans ses multiples expressions participatives, notamment dans ce qu'il donne à voir de lui au travers des processus de mise en durabilité de la ville et de ses quartiers. Les écoquartiers sont à ce titre des révélateurs de mutations à l'œuvre. Espaces qui condensent l'ensemble des enjeux socio-urbains et politiques du moment, ils prétendent aussi organiser les comportements jusqu'à saisir l'individu dans le privé et dans l'intime. Mais c'est sans doute en ce que ces projets demandent une implication personnelle plus forte, souvent sur le mode de l'injonction et parfois de la contrainte, qu'ils deviennent perméables à des réactions d'autonomisation participative, à des modes d'implication comme modes de vie, plus individués, donc parfois plus discrets mais tout autant « reliants » et résistants, tels que les donnent aussi à voir ce dossier. Pour autant, les potentiels d'innovations sociales participatives ne s'épanouissent pas sans frottements, notamment face à un « nouvel esprit de la ville » (Pattaroni 2011) qui, tel que souvent promu par les instances politiques, s'accompagne souvent d'un véritable durcissement des politiques urbaines à l'égard des formes de vie alternatives, des appropriations marginales, mais aussi d'une résorption progressive des lieux et des ressorts d'une pensée vive de l'utopie qui sont la condition même d'une invention de nouveaux possibles participatifs.

\section{BIBLIOGRAPHIE}

Bertheleu H., Neveu C., 2006, « De petits lieux du politique : individus et collectifs dans des instances de « débat public » à Tours. », Espaces et sociétés 1/2006 (nº 123), p. 37-51.

Bonard Y., Matthey L., 2010, « Les éco-quartiers : laboratoires de la ville durable ? ", Cybergéo : European Journal of Geography [en ligne], Débats, Quartier durable ou éco-quartier? Mis en ligne le 9 juillet 2010.

Emelianoff C., 2008, « Enjeux et figures d'un tournant urbanistique en Europe, Responsabilité et environnement », Annales des Mines, ${ }^{\circ}$ 2, octobre, p. 15-20.

Gourgues G., 2013, Les politiques de démocratie participative, PUG, 148 p.

Leresche J.-P., Audétat M. (dir.), 2006. « Participation et développement urbain durable », Urbia, n $\circ 3$. 
Mathieu N., Guermond N., 2011, La ville durable, du politique au scientifique, Quae, 285 p.

Mermet L., 2007, « Débattre, sans savoir pourquoi : la polychrésie du débat public appelle le pluralisme théorique de la part des chercheurs ", in M. Revel, C. Blatrix, L. Blondiaux et al., Le débat public : une expérience française de démocratie participative, La Découverte, $412 \mathrm{p}$.

Pattaroni L., 2011, « Le nouvel esprit de la ville. Les luttes urbaines sont-elles recyclables dans le «développement urbain durable »? ", Mouvements, $n^{\circ}$ 65, janvier-mars, p. 43-56

Schaeffer V., Ruegg J., Litzistorf-Spina N., 2010, « Quartiers durables en Europe : enjeux sociaux et processuels ", Urbanisme, n³71, p. 27-32.

Scott James C., 2006, « Infra-politique des groupes subalternes », Vacarme, n 36, été, p. 25-29.

\section{INDEX}

Keywords : citizen participation, ecodistricts, politicization, sustainable urban planning Mots-clés : participation habitante, écoquartiers, politisation, ville durable

\section{AUTEURS}

\section{PASCAL TOZZI}

Pascal Tozzi, UMR ADESS 5185, a été responsable du programme ANR « ville durable » PAGODE (2010-2014). Il a récemment coordonné l'ouvrage : L'animation socioculturelle, quelle place dans le projet urbain? (Carrières sociales éditions, 2014), pascal.tozzi@cnrs.fr

\section{ABDOURAHMANE NDIAYE}

Abdourahmane Ndiaye, UMR ADESS 5185, a été le secrétaire scientifique du programme ANR « ville durable » PAGODE (2011-2014). Il a dirigé l'ouvrage : Économie sociale et solidaire: Animation et dynamiques des territoires (L'Harmattan, 2011), ndiaye.a@ades.cnrs.fr

\section{AURÉLIE CARIMENTRAND}

Aurélie Carimentrand, UMR ADESS 5185, est maître de conférences en sciences économiques à l'IUT Bordeaux Montaigne. Elle travaille depuis une dizaine d'années sur des thèmes relatifs à la consommation responsable et à la réappropriation citoyenne de l'économie, aurelie.carimentrand@iut.u-bordeaux-montaigne.fr 